\title{
Conductor grid optimization for luminance loss reduction in organic light emitting diodes
}

\author{
Kristiaan Neyts, a) Alfonso Real, Matthias Marescaux, Saso Mladenovski, and \\ Jeroen Beeckman \\ ELIS, Ghent University, Sint-Pietersnieuwstraat 41, B-9000 Gent, Belgium
}

(Received 23 October 2007; accepted 18 February 2008; published online 12 May 2008)

The recently increased efficiency of organic light emitting devices (OLED) brings lighting applications within reach. If the area of the OLED is of the order $\mathrm{a} \mathrm{cm}^{2}$, voltage and brightness losses related to the square resistance of the transparent electrode become important. The homogeneity of the voltage and brightness can be improved by contacting the transparent electrode from all edges and by adding a metallic grid to the transparent electrode. This grid should have narrow lines to minimize transmission losses and improve the total light emission from the OLED. The voltage losses in grids with different shapes (triangular, square, and hexagonal) are evaluated and the grid parameters are optimized to maximize the total light emission. It turns out that a hexagonal grid has lower loss than a square grid with the same line width. (c) 2008 American Institute of Physics. [DOI: 10.1063/1.2907960]

\section{INTRODUCTION}

As white organic light emitting diodes (OLEDs) reach efficiencies in the range of $50 \mathrm{~lm} / \mathrm{W}$, large area lighting applications come into reach. The resistivity of conventional transparent electrodes sets serious limitations to the maximum size of the OLED, because there is a voltage and luminance drop related to the current flowing laterally in the transparent electrode. ${ }^{1}$ For commercial transparent electrode layers, the losses become important for devices with dimensions in the order of a few centimeters, so it is essential to provide a metallic grid in contact with the transparent electrode. The problem of voltage loss and grid optimization has also been investigated for solar cells. ${ }^{2-6}$

This paper introduces a numerical model for the voltage loss in the OLED anode which is in contact with a metallic grid and uses this model to compare different regular grids. The aim is to find an expression for the different loss mechanisms that occur in an OLED with metallic grid (voltage loss in the grid, voltage loss in the electrode, and transmission loss) and to use this to optimize the grid parameters.

\section{MODELING THE GRID}

For the electrical modeling of the OLED with the conductor grid we consider three conducting layers (see Fig. 1): a metallic grid (not homogeneous), a transparent bottom electrode, and a grounded metallic top electrode (reflective), with respective thicknesses $d_{g}, d_{b}$, and $d_{t}$. The thicknesses of these layers (order of $1 \mu \mathrm{m}$ ) are much smaller than the lateral dimensions of the OLED device (order of $1 \mathrm{~mm}$ ) and we can make the following approximations:

- The cathode can be considered as a perfect conductor and it is grounded: $V_{t}=0$.

- The organic layers have a much higher resistivity than

${ }^{a)}$ Electronic mail: kristiaan.neyts@elis.ugent.be. the bottom electrode, therefore the potential in the bottom electrode is independent of the $z$-coordinate $V_{b}(x, y)$.

- For the same reason, the grid electrode potential is equal to the bottom electrode potential: $V_{g}(x, y)$ $=V_{b}(x, y)$.

- As in Ref. 1, we assume that the current density $\left(\mathrm{A} / \mathrm{m}^{2}\right)$ in the emitting layer is in first approximation homogeneous and perpendicular to the substrate: $j_{\mathrm{Z}}$. This is acceptable because in a realistic design the relative brightness variations should be limited to about $10 \%$.

The lateral current density in a conducting layer is proportional with the electric field and the material conductivity $\sigma$

$$
\begin{aligned}
& \vec{j}_{b}=-\sigma_{b} \operatorname{grad} V_{b}, \\
& \vec{j}_{g}=-\sigma_{g} \operatorname{grad} V_{g} .
\end{aligned}
$$

The conservation of the current flux in a stationary situation requires

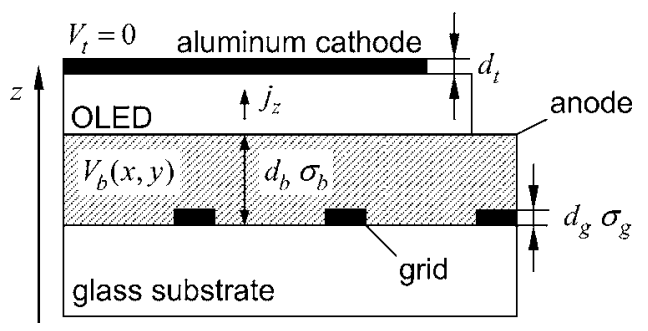

FIG. 1. Cross-section of OLED device with aluminum cathode and transparent bottom electrode. Below the anode a metallic grid is applied. The thicknesses and conductivities of the different layers are indicated. 

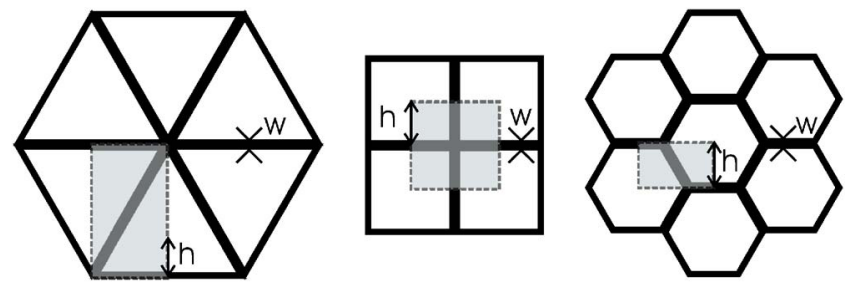

FIG. 2. (Color online) Top view of the three regular metallic grids: triangular (left), square (middle), and hexagonal (right). The apothem $h$ and the line width $w$ are the same for the three grids. For each grid an elementary rectangular building block is indicated in the figure.

$$
\operatorname{div}\left(d_{b} \vec{j}_{b}+d_{g} \vec{j}_{g}\right)=-j_{z} \quad \text { or } \operatorname{div}\left(d_{b} \vec{j}_{b}\right)=-j_{z},
$$

for regions with or without the presence of the metal grid, respectively. Eliminating the current densities $j_{z}$ from these equations leads to the following differential equation for the electrode voltages, again in two regions:

$$
\nabla^{2} V_{b}=\frac{R_{b} R_{g}}{R_{b}+R_{g}} j_{z} \approx R_{g} j_{z} \quad \text { or } \nabla^{2} V_{b}=R_{b} j_{z},
$$

with $R(\Omega)$ the sheet resistance of the electrode,

$$
R_{b, g}=\frac{1}{\sigma_{b, g} d_{b, g}},
$$

and $R_{g} \ll R_{b}$. This means that we can model the combination of the bottom electrode and grid as a single electrode with inhomogeneous sheet resistivity $R_{b}$ when there is no grid and $R_{g}$ when there is a metal grid below the bottom electrode. In short notation,

$$
\nabla^{2} V_{b}(x, y)=R(x, y) j_{z} .
$$

In this paper we consider three types of regular grids: triangular, square, and hexagonal, as illustrated in Fig. 2. We characterize the grid by the apothem $h$ of the holes in the grid and the width $w$ of the metal lines (see Fig. 2). Usually the features of the metal grid are much smaller than the total dimensions of the grid, and therefore it is interesting to determine the macroscopic properties of the grid. Due to the three- or fourfold rotation symmetries of the grid structures, the macroscopic resistivity tensor reduces to a scalar effective sheet resistivity. By evaluating the current for a given voltage difference over an elementary rectangle of the grid, and assuming $R_{g} \ll R_{b}$ and $w \ll h$, the effective sheet resistivities of the three grids can be determined as

$$
R_{\mathrm{eff}} \approx \frac{2 h}{w} R_{g} .
$$

This is easily verified for the square grid, by considering an elementary square as in Fig. 2 . For a field in the $x$-direction, for example, only the horizontal line contributes to conduction and its resistance in the elementary square is given by Eq. (6). The same formula (6) is found for triangular and hexagonal grids by considering the grid resistance in the horizontal direction [respectively, $2 h / \sqrt{3} w\left(R_{g}\right)$ and $\left.2 \sqrt{3 h} / w\left(R_{g}\right)\right]$ and the length/width ratio (respectively, $1 / \sqrt{3}$ and $\sqrt{3}$ ) for the elementary rectangles that are indicated in Fig. 2.
TABLE I. Number of sides, element area, and geometry factor $f$ for the three grid element shapes and for a circular element.

\begin{tabular}{lccc}
\hline \hline \multicolumn{1}{c}{ Element shape } & No. of sides & Element area & Geometry factor $f$ \\
\hline Triangular & 3 & $3 \sqrt{3} h^{2}$ & $0.152 \pm 0.001$ \\
Square & 4 & $4 h^{2}$ & $0.143 \pm 0.001$ \\
Hexagonal & 6 & $2 \sqrt{3} h^{2}$ & $0.134 \pm 0.001$ \\
Circular & $\infty$ & $\pi h^{2}$ & 0.125 \\
\hline \hline
\end{tabular}

As the bottom electrode is transparent and the metallic grid is not, the transmissivity $T$ of the grid is equal to the area of the metal grid divided by the total area,

$$
T=\left(\frac{h}{h+w / 2}\right)^{2} \approx 1-\frac{w}{h},
$$

with the approximation holding for $w \ll h$. The two previous equations indicate that grids with the same parameters $w$ and $h$ have the same effective resistivity and transmissivity. ${ }^{7} \mathrm{We}$ will now evaluate if they are also equivalent with respect to the voltage loss inside a grid element.

\section{AVERAGE VOLTAGE INSIDE A GRID ELEMENT}

In this section we determine the voltage loss inside a grid element, assuming that the voltage on the metal grid is constant and equal to $V_{g}$. Because the metal grid is typically much more conductive than the transparent electrode, this is usually a good assumption. Inside a polygon grid element, the potential of the bottom electrode is described by the differential equation (5) with $R=R_{b}$. For many applications the function that has to be optimized is proportional with the voltage or can be linearized in a small voltage interval, e.g., the OLED brightness. Therefore it is important to maximize the average voltage over a grid element (or to minimize the average voltage loss). A dimension analysis of the differential equation shows that the voltage loss is proportional with $R_{b}, j_{z}$, and $h^{2}$ and therefore, we can write

$$
V_{b, \text { avg }}=V_{g}-f_{\text {shape }} R_{b} j_{z} h^{2},
$$

with $f_{\text {shape }}$ a dimensionless geometry factor depending on the shape of the grid element (triangular, square, or hexagonal). For each shape, we solved the differential equation (5) for $V_{b}$ with a finite difference method, fixing the given voltage $V_{g}$ on the edges of the element. From the average voltage $V_{b \text {,avg }}$ inside the element, the geometry factor $f_{\text {shape }}$ can be found from Eq. (8) for the three shapes and the results are presented in Table I. This shows that the voltage loss in a square element is about $6 \%$ larger than the loss in a hexagonal element, for equivalent grids with the same apothem $h$. For a circular element the apothem $h$ is equal to the radius and the geometry factor can be calculated analytically as a function of the distance from the center $r$ (see Ref. 1),

$$
V_{b}(r)=V_{g}-R_{b} j_{z} \frac{h^{2}-r^{2}}{4},
$$

yielding $f_{\text {circle }}=0.125$ after averaging. From the finite difference simulation we find a value of 0.126 , indicating that the 

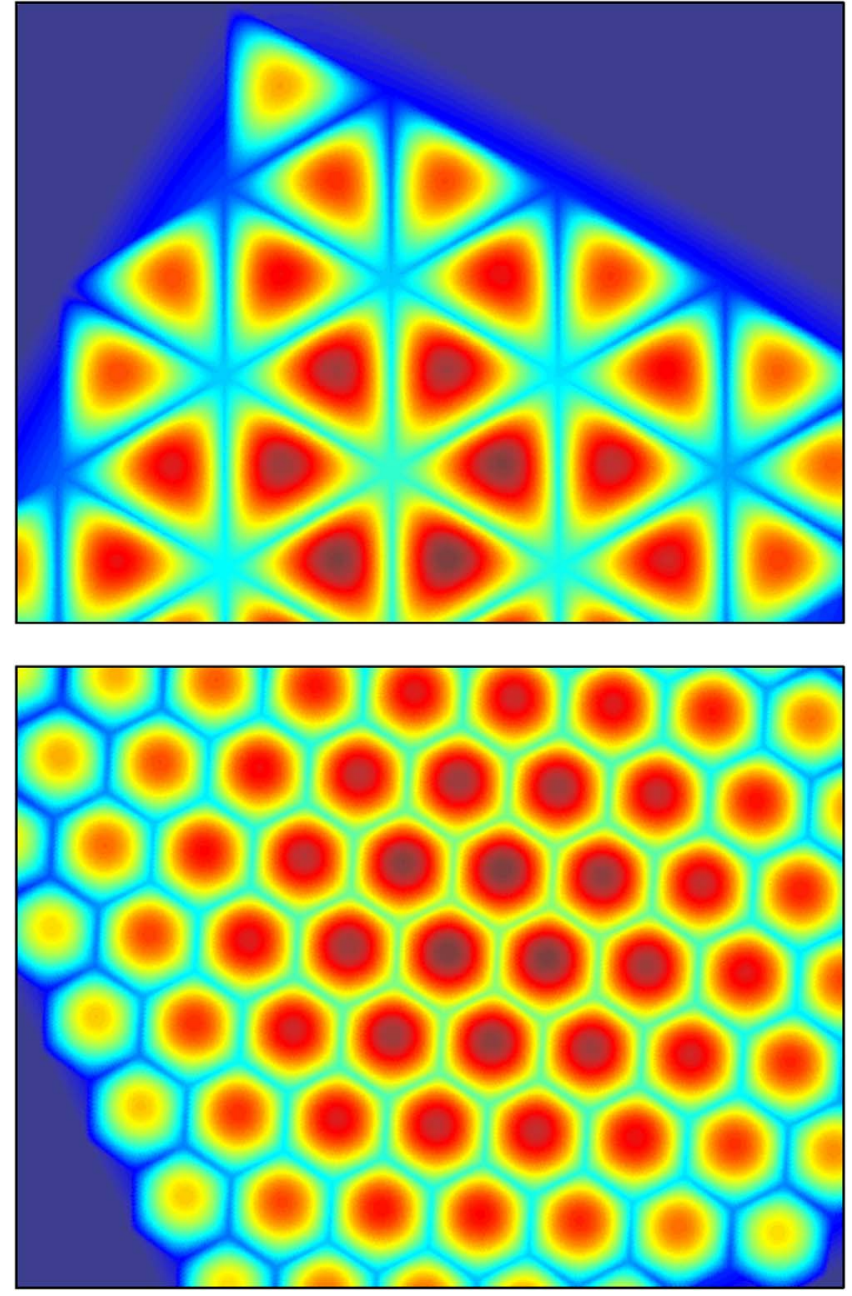

FIG. 3. (Color online) Simulated lateral variation of the electrode voltage $V_{b}(x, y)$ for a square OLED device with two different metal grids: triangular grid (top) and hexagonal grid (bottom).

accuracy of the simulation results in Table I is of the order of $1 \%$.

\section{OPTIMIZING THE GRID PARAMETERS}

We now consider a square OLED panel with side $s$, with a metal grid below the bottom electrode and the four edges of the square held at a certain potential $V_{\text {edge }}$. The voltage $V_{b}(x, y)$ can be determined numerically by using the differential equation (5). Examples of electrode potentials for triangular and hexagonal grids are given in Fig. 3. For large structures the parameter simulation and parameter optimization of a certain design require a lot of calculation time. Therefore we will follow an alternative approach, using a few simplifying assumptions.

The average voltage in the metal grid (averaged over the total area $s^{2}$ of the device) can be determined by applying the same theory as in the previous section (using $R_{\text {eff }}$ instead of $R_{b}$ ), and we find

$$
V_{g, \text { avg }}=V_{\text {edge }}-\frac{1}{4} f_{\text {square }} R_{\text {eff }} j_{z} s^{2} .
$$

The voltage inside the grid elements is lower, because there are also voltage losses in the transparent electrode. To obtain

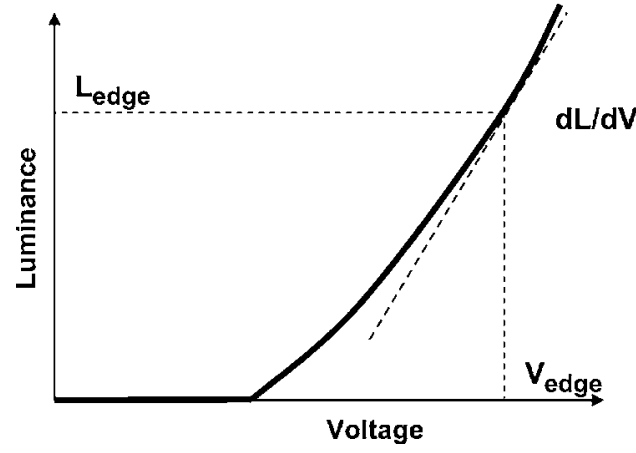

FIG. 4. Schematic luminance-voltage characteristic of an OLED. As the lateral variation in the luminance should remain limited, it is a good approximation to use the derivative (dashed line) in the modeling.

the average potential of the transparent electrode both loss mechanisms can be added if they are sufficiently small. Using Eqs. (8) and (10), this yields

$$
V_{b, \text { avg }}=V_{\text {edge }}-f_{\text {shape }} R_{b} j_{z} h^{2}-\frac{h}{2 w} f_{\text {square }} R_{g} j_{z} s^{2} .
$$

Let us now consider the case of an OLED for which the brightness is a linear function of the voltage (Fig. 4). As small variations in the luminance are targeted, such a linear variation is an acceptable assumption.

The presence of the metal grid reduces voltage (and brightness) losses, but there is no light emitted from below the grid, because the metal is absorbing. We assume that the width of the gridlines is large compared to the wavelength, so outcoupling effects can be neglected. Therefore, we can consider the transmission of Eq. (7) as an additional loss mechanism and write for the average brightness of the device

$$
\begin{aligned}
\frac{L_{\text {avg }}}{L_{\text {edge }}}= & \left(1-\frac{j_{z}}{L_{\text {edge }}} \frac{d L}{d V}\left(f_{\text {shape }} R_{b} h^{2}+f_{\text {square }} R_{g} \frac{h s^{2}}{2 w}\right)\right) \\
& \times\left(1-\frac{w}{h}\right) \approx 1-\alpha f_{\text {shape }} \frac{h^{2}}{w^{2}}-\frac{1}{2} \gamma f_{\text {square }} \frac{h}{w}-\frac{w}{h},
\end{aligned}
$$

with $d L / d V$ as the slope of the $L(V)$ characteristic and

$$
\begin{gathered}
\alpha=\frac{R_{b} j_{z} w^{2}}{L_{\text {edge }}} \frac{d L}{d V}, \\
\gamma=\frac{R_{g} j_{z} s^{2}}{L_{\text {edge }}} \frac{d L}{d V},
\end{gathered}
$$

which are two dimensionless parameters. The three loss terms are related to, respectively, the loss in a grid element, the loss in the grid, and the loss in transmission. In the case that the area above the metallic grid would not be active (not drawing any current), another weight factor should be used for the transmission loss.

Maximizing Eq. (12) with $w$ and $h$ as free parameters leads to an infinitely fine grid ( $w$ and $h$ zero) which cannot be realized technologically. Usually the technology sets a lower limit for the width $w$ of the gridlines and we will use this value in our optimization. With $w$ given, we can find the overall optimum value $h^{*}$ that maximizes the average lumi- 

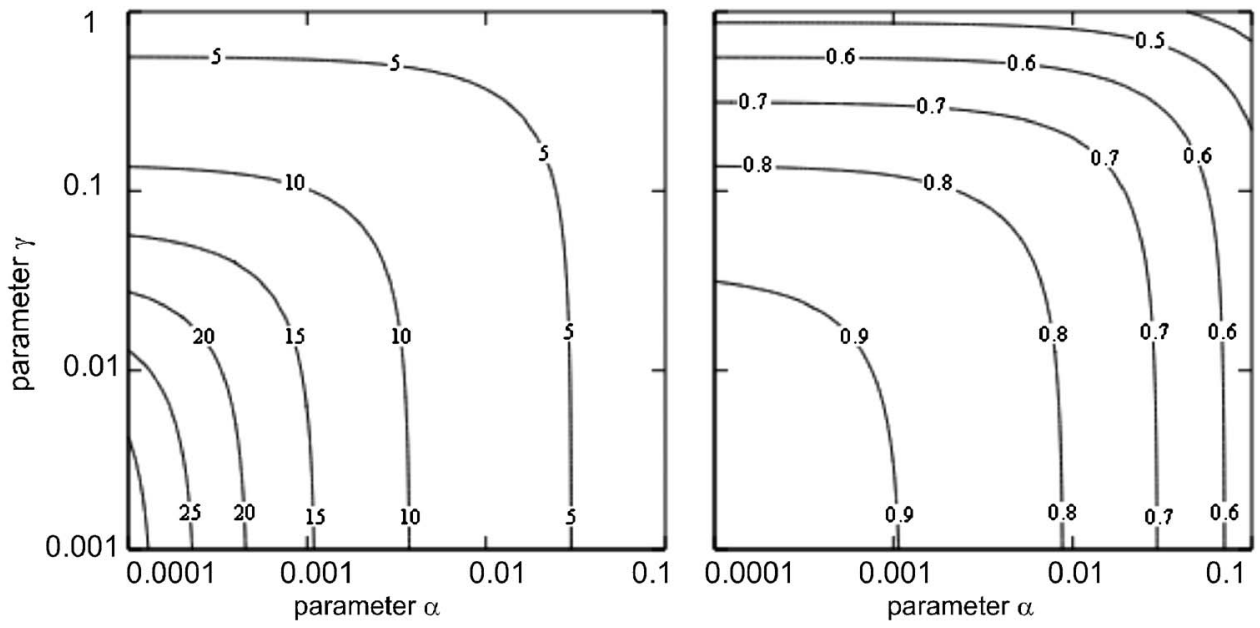

FIG. 5. Optimal value for $h^{*} / w$ (left) and the corresponding relative average luminance $L_{\text {avg }} / L_{\text {edge }}$ (right) as a function of the two dimensionless parameters $\alpha$ and $\gamma$. nance of formula (12) by solving a third order polynomial equation. First we consider two particular cases for which one of the loss terms can be neglected: the loss in the grid or the loss in the holes, respectively.

For the case that the loss in the grid [second loss term in Eq. (12)] can be neglected, the optimum $h^{*}$ is determined by the parameters of one grid element,

$$
\frac{h_{\text {shape }}^{*}}{w}=\sqrt[3]{\frac{1}{2 f_{\text {shape }} \alpha}} .
$$

For this optimum value, the loss in the grid element (first term) is half of the transmission loss (third term). If the relative loss in average luminance should be smaller than $\varepsilon$, then $\alpha$ should satisfy

$$
\alpha<\frac{4 \varepsilon^{3}}{27 f_{\text {shape }}} .
$$

For a luminance $\operatorname{loss} \varepsilon=0.1$ and a grid with hexagonal shape the maximum value for $\alpha$ is 0.0011 . For a given OLED, with a voltage operation point and an electrode width, the maximum sheet conductivity of the bottom electrode is given by

$$
R_{b}<\frac{4 \varepsilon^{3} L_{\text {edge }}}{27 f_{\text {shape } j_{z} w^{2}}} \frac{d V}{d L} .
$$

For the case that the loss in the grid element [first loss term in Eq. (12)] can be neglected, the optimum $h^{*}$ is determined by the parameters of the grid,

$$
\frac{h_{\text {grid }}^{*}}{w}=\sqrt{\frac{2}{f_{\text {square }} \gamma}} .
$$

For this optimum value, the loss in the grid (second term) equals the transmission loss (third term). If the relative loss in average luminance should be smaller than $\varepsilon$, then $\gamma$ should satisfy

$$
\gamma \leqslant \frac{\varepsilon^{2}}{2 f_{\text {square }}} .
$$

For a luminance loss $\varepsilon=0.1$ and the maximum value for $\gamma$ is 0.035 . For a given OLED, with a voltage operation point and an electrode width, the maximum sheet conductivity of the bottom electrode is given by

$$
R_{g} \leqslant \frac{\varepsilon^{2} L_{\text {edge }}}{2 f_{\text {square }} j_{z} s^{2}} \frac{d V}{d L} .
$$

The general solution for $h^{*}$ (when all three terms are important) can be found by solving a third order polynomial equation. The solution as a function of the parameters $\alpha$ and $\gamma$ is given in Fig. 5. Small values of $\alpha$ and $\gamma$ allow to use a grid with a larger apothem and this result in a higher average brightness. It can be seen from this figure that the global minimum $h^{*}$ is smaller than the optimal values calculated for the two simplified cases,

$$
h^{*}<\min \left(h_{\text {shape }}^{*}, h_{\text {grid }}^{*}\right) \text {. }
$$

\section{OPTIMIZATION EXAMPLES}

Consider an OLED device which is driven at the following operation point, as in Ref 1:

$$
\begin{aligned}
& V_{\text {edge }}=3.9 \mathrm{~V}, \\
& L_{\text {edge }}=1200 \mathrm{Cd} \mathrm{m}^{-2}, \\
& j_{z}=50 \mathrm{~A} \mathrm{~m}^{-2}, \\
& \frac{d L}{d V}=2000 \mathrm{Cd} \mathrm{m}^{-2} \mathrm{~V}^{-1} .
\end{aligned}
$$

We will now consider different electrode/grid structures and investigate the dimensions for which an average luminance loss is as small as possible.

The first structure is a square device without grid and with fixed voltage $V_{\text {edge }}$ at the edges. For the bottom electrode we assume a sheet resistance of $R_{b}=200 \Omega$, corresponding to the spin-coated polymer conductor Baytron. ${ }^{1,8}$ In this case only the first loss term in (12) is important and we find that the value for the apothem (half of the side) that realizes a loss $\varepsilon=10 \%$ is given by 


$$
h=\sqrt{\frac{L_{\text {edge }}}{f_{\text {square }} R_{b} j_{z} \varepsilon d L / d V}}=65 \mathrm{~mm} .
$$

Using an ITO electrode with a sheet resistance of only $20 \Omega$ allows to enlarge the apothem of the square to $205 \mathrm{~mm}$, keeping the same $10 \%$ luminance loss.

The second structure is a square panel with side $s$ $=0.5 \mathrm{~m}$ using an aluminium grid with $\sigma_{g}=42$ $\times 10^{6} \Omega^{-1} \mathrm{~m}^{-1}, w=1 \mathrm{~mm}$, and $d_{g}=10 \mu \mathrm{m}$. In this case the square resistivity of the grid material is only $0.0024 \Omega$ and the parameters $\alpha$ and $\gamma$ have the values 0.017 and 0.05 , respectively. For the hexagonal grids the optimum value for the apothem is in this case $5.8 \mathrm{~mm}$ and the average luminance is $73.2 \%$ of the luminance near the edge. For the square and triangular grids, the optimal apothems are, respectively, 5.7 and $5.6 \mathrm{~mm}$ and the average relative luminances are $72.7 \%$ and $72.2 \%$.

\section{CONCLUSION}

In this paper, we studied the voltage variation in a transparent electrode, containing a regular metallic grid, of an OLED. The differential equation was used to determine the average voltage loss in grid elements with different shapes: triangular, square, or hexagonal. It is found that grids with identical line width and apothem have the same conductivity and transmissivity, For such equivalent grids, the hexagonal grid has the lowest average voltage loss, which is $6 \%$ lower than for the square grid. An approximate analytical expression for the different loss mechanisms (loss in the grid, loss in the grid elements, and loss in transmission) is developed and an optimization algorithm for the apothem, using a fixed electrode width, is described. The results provide a simple means to estimate and optimize the different loss mechanisms in OLEDs with a metallic grid below the transparent conductor.

\section{ACKNOWLEDGMENTS}

The authors would like to acknowledge the support of the European Commission through the sixth framework Integrated Project OLLA - Organic LED's for Lighting Applications and the support of the Belgian Science Policy project P6/10 photonics@be. Jeroen Beeckman is a postdoctoral fellow of the Research Foundation Flanders (FWO).

${ }^{1}$ K. Neyts, M. Marescaux, A. Ullan Nieto, A. Elschner, W. Loevenich, K. Fehse, Q. Huang, K. Walzer, and K. Leo, J. Appl. Phys. 100, 114513 (2006).

${ }^{2}$ A. Cheknane, B. Benyoucef, J. P. Charles, R. Zerdoum, and M. Trari, Sol. Energy Mater. Sol. Cells 87, 557 (2005).

${ }^{3}$ R. Ebner, M. Radike, V. Schlosser, and J. Summhammer, Prog. Photovoltaics 11, 1 (2003).

${ }^{4}$ A. Luque, Solar Cells and Optics for Photovoltaic Concentration, The Adam Hilger Series on Optics and Optoelectronics, edited by E. R. Pike and W. T. Welford (Adam Hilger, Bristol, 1989).

${ }^{5}$ T. Aernouts, P. Vanlaeke, W. Geens, J. Poortmans, P. Heremans, S. Borghs, R. Mertens, R. Andriessen, and L. Leenders, Thin Solid Films 451-452, 22 (2004).

${ }^{6}$ G. P. Kushto, W. Kim, and Z. H. Kafafi, Appl. Phys. Lett. 86, 093502 (2005).

${ }^{7}$ G. A. H. M. Vrijssen and A. A. Van Gorkum, U.S. Patent No. 4,684,994 (1987).

${ }^{8}$ Baytron ${ }^{\circledR} \mathrm{P}$ is a waterborne dispersion of poly(ethylendioxythiophene) (PEDOT) and Poly(styrenicacid) (PSS); see datasheets at www.baytron.com. 
Journal of Applied Physics is copyrighted by the American Institute of Physics (AIP). Redistribution of journal material is subject to the AIP online journal license and/or AIP copyright. For more information, see http://ojps.aip.org/japo/japcr/jsp 